\title{
Oral Mucormycosis in an Allogeneic Stem Cell Transplant Recipient
}

\author{
Marchese $\mathbf{M L}^{1}$, Ruiz Beguerie $\mathbf{J}^{1^{*}}$, Fernandez $\mathbf{A}^{1}$, Anaya $\mathbf{J}^{2}$, and Valdez $\mathbf{R}^{1}$ \\ ${ }^{1}$ Dermatology Department, Stomatology Service, Austral University Hospital, Argentina \\ ${ }^{2}$ Pathology Department, Austral University Hospital, Argentina
}

"Corresponding author: Ruiz Beguerie J, Dermatology Department, Austral University Hospital, Austral University, Av.Juan Domingo Perón 1500, Buenos Aires Province 1629, Argentina, Tel: +54.1230.448.2000; E-mail: jruiz@cas.austral.edu.ar

Received date: Jun 13, 2016; Accepted date: Jul 30, 2016; Published date: Aug 3, 2016

Copyright: (c) 2016 Marchese ML, et al. This is an open-access article distributed under the terms of the Creative Commons Attribution License, which permits unrestricted use, distribution, and reproduction in any medium, provided the original author and source are credited.

\begin{abstract}
Oral mucormycosis is an unusual and a lethal infection in patients following hematopoietic stem cell transplantation. A 20 year old man was diagnosed at the Austral University Hospital with palatal mucormycosis on day +20 after a hematopoietic stem cell transplant because of an acute leukemia lymphoblastic. There are less than 25 palatal mucormycosis cases reported in the literature. This case highlights the importance of being aware of this uncommon, but potentially fatal condition when assessing patients with transplants for a prompt diagnosis and intervention.
\end{abstract}

Keywords: Oral mucormycosis; Hematopoietic stem cell transplant; Allogeneic stem cell transplant; Immunocompromised patients; Hematological disease; Acute lymphoblastic leukemia; Nodular lesion

\section{Introduction}

Mucormycosis is a deep fungal infection due to a fungus of the Mucorales species. Most human infections of these causative fungi include species such Rhizopus, Cunninghamella, Rhizomucor, Mucor, and Absidia spp. Mucorales are ubiquitous filamentous fungi, with a variable width, non-septate or sparsely septate [1]. They are present in environments such as soil, bread mould, decaying vegetation and dust, and their infectious spores are initially inhaled which can establish a route of infection in the sinuses. Other routes include ingestion through the digestive tract or inoculation through breaches in the skin [2]. These fungi are commonly non-pathogenic in healthy people, they are normally found in the nasal mucosa of the immune competent individual, but may become opportunistic pathogens in immunecompromised patients associating this infection with high rates of morbidity and mortality. Conditions that predispose to infection include diabetes, long-term steroid medication, hematologic malignancies, solid organ and hematologic stem cell transplantation (SCT). In addition, iron overload plays an important role in the growth of mucormycosis [3]. The most common sites of mucormycosis are pulmonary, sinus, nasal, skin and disseminated infections. More than one third of all cases are a rhinocerebral infection starting in the nasal sinuses and spreading to the face, palate, orbits and brain [4].

In the past decades, mucormycosis has emerged as an important invasive fungal infection in stem cell transplant recipients, in whom it often has an aggressive clinical course and a high mortality. Several risk factors may be found in this population, although the potent $\mathrm{T}$ cell depleting drugs and/or antibodies used for immunosuppression in transplant recipients are responsible for the high-risk of invasive fungal infection in this group [5].

Mucormycosis is the third most common fungal disease in SCT after candidiasis and aspergillosis. In a follow-up of 1,500 SCT patients, mucormycosis was diagnosed in $0.9 \%$ of them with a mortality of $77 \%$ [6]. The most common oral sign of mucormycosis is necrosis or ulceration of the hard palate that can result in hematogenous spread leading to fulminant infection and death.

We report the case of a patient who was under immunosuppression due to treatment because of his hematological disease. Early diagnosis, resolution of immunosuppression and appropriate antifungal treatment is essential to achieve a good outcome.

\section{Case Report}

A 20 year old man with acute lymphoblastic leukemia in remission received an

unrelated allogeneic stem cell transplant. He was conditioned with clofarabinebusulfan-antitimoglobulin and was under prophylaxis with tacrolimus and methotrexate. He presented infectious complications such as reactivation of cytomegalovirus (CMV) and a catheter infection with Candida parapsilosis. On day +20 the Internal Medicine team requested a consultation to the Dermatology Department because of a tissue injury of the hard palate that had appeared 24 hours ago, not painful. The patient was under antibiotic treatment with Piperacilin-Tazobactam (PTZ) because of febrile neutropenia.

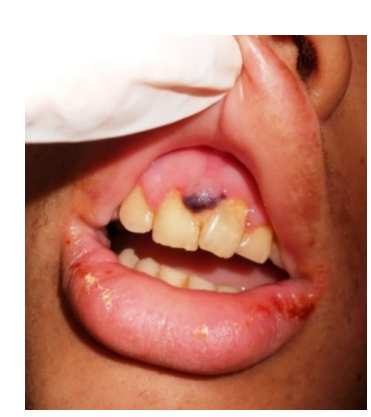

Figure 1: An ulcerated, exophytic, nodular lesion with necrotic areas in front of central incisors. 
Examination revealed an ulcerated, exophytic, nodular lesion with necrotic areas and local tenderness on palpation of about $1 \times 1.5 \mathrm{~cm}$. in front of central incisors (Figure 1). No other sites of ulceration or necrosis were noted. The dermatology team arrived at a differential diagnosis of mucormycosis because of the necrosis area on the hard palatal. A biopsy was performed and the sample was sent for microbiological and for histopathological examination.

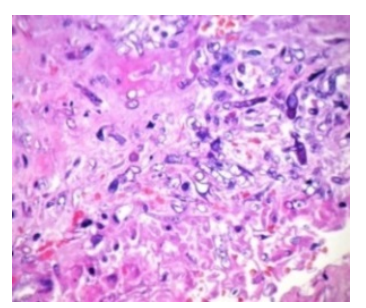

Figure 2: Fungal filaments some branched and spores (H-E. 40x).

Computed tomography (CT) of mandible with intravenous contrast suggested no bone or sinus involvement. The CT chest found a diffuse infiltrate in frosted glass. Mucor racemosus was confirmed in the microbiological sample and fungal filaments, some branched and spores (Figure 2) were observed in the histopathology with hematoxylin and eosin (H-E) sample. Periodic acid-Schiff (PAS) and Grocott staining confirmed fungal filaments (Figure 3).

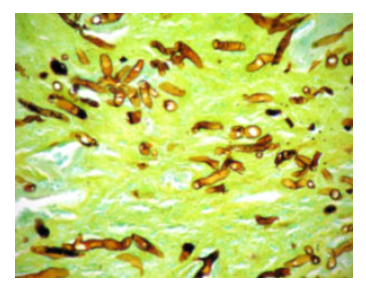

Figure 3: Grocott staining: fungal filaments.

Treatment was initiated with liposomal amphotericin B (L-Amb) 5 $\mathrm{mg} / \mathrm{kg} /$ day and the maxillectomy with wide toilette of soft tissue and the extraction of the two central incisors was performed by the Head and Neck Surgery team.

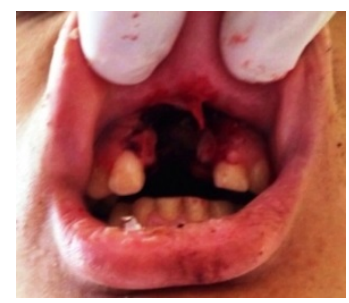

Figure 4: Surgical toilette with extensive bone and incisors resection.

He evolved with superinfection by Acinetobacter baumannii and progression of oral mucormycosis with sinus and pulmonary disease requiring multiple surgical toilettes with extensive bone resection (Figure 4) and high LAmb doses to $7 \mathrm{mg} / \mathrm{kg} /$ day. He presented engraftment failure with severe and prolonged neutropenia with daily platelets, red blood cells and granulocytes transfusions. Under a great immunosuppression the patient developed a septic shock with multiorgan failure and death.

\section{Discussion}

Oral mucormycosis is a rare infection primarily seen in immunecompromised patients mainly reported in the literature as case reports. Most cases are associated to mucormycosis in transplant recipients. Results are difficult to compare because of the lack of uniform diagnostic criteria and the geographical variation in exposures [7]. In addition, the poor autopsy data from transplant recipients makes it difficult to define and generate consensus between physicians. Intraoral presentation is most commonly reported on the hard palate leading to tissue necrosis presenting with ulceration and bone destruction leading to the loosening of the teeth [8]. The property of the disease is the facility to cause angioinvasion, which results in thrombosis and in tissue necrosis. This contributes to the capacity of the organism to rapidly disseminate into the blood and to other organs, such as the brain or the lungs leading to disseminated sepsis [9].

Cases of oral mucormycosis have recently been published in the Medical Mycology Journal [10]. The final diagnosis is usually made by culture and treatments include local surgical debridement involving extraction of adjacent teeth and bone resection and amphotericin B, which lead to resolution in most cases. Six other cases were identified in the literature, three involving gingival sites and three developed following a dental extraction. Treatment in these cases included locoregional surgery and amphotericin B [5]. Successful management depends on the recognition of patients at risk searching for the early signs of infection; removal or reduction of risk factors such as the level of immunosuppression by administering tapering doses of steroids and immunosuppressive agents, controlling hyperglycemia and providing appropriate antifungal treatment and surgical debridement, which improve survival.

Amphotericin B still is widely considered as the treatment of choice. The 2009 recommendation guide from the European Conference on Infections in Leukemia advocates the use of a lipid formulation of amphotericin B as first-line therapy for mucormycosis [11]. Most experts use liposomal amphotericin B with a starting dose of $5 \mathrm{mg} / \mathrm{kg}$ / day, which is sometimes increase to $10 \mathrm{mg} / \mathrm{kg} /$ day, if needed, over a period of 6-12 weeks [10]. However, more recently, posaconazole (800 $\mathrm{mg}$ /day) has been used after reports of success from individual cases, or a combination of the 2 in refractory cases [12]. Actually we know that mucormycosis represents $8 \%$ of invasive fungal infections in SCT recipients [3], so common risk factors, diagnostic strategies and treatment for mucormycosis should be known by physicians in transplant and infectious diseases units.

The administration of a lipid formulation of amphotericin B and, when reasonable, surgery also, will optimize the outcome for patients with mucormycosis in the transplant situation $[13,14]$. The value of reversing underlying factors is evident in the experience with mucormycosis in transplant recipients. Neutrophil recuperation is extremely significant because neutrophils play an important role in the host defense against Mucorales [15].

Adjunctive therapies for mucormycosis in the literature suggest the use of hyperbaric oxygen therapy and iron chelating agents that improve the prognosis of this aggressive disease. Hyperbaric oxygen inhibits fungal growth and promotes the action of amphotericin B. It 
also promotes tissue healing by enhancing the immune response and improves tissue oxygenation. A review of the literature showed that patients receiving hyperbaric oxygen therapy had higher survival rates provided that the underlying condition is reversed. Iron chelation is reported to improve the prognosis, as iron is important for fungal growth [16].

In conclusion, a high index of suspicion and careful clinical and radiologic examinations are the key to early identification of probably infected patients. In most severely ill neutropenic patients, only aggressive antifungal therapy together with immune reconstitution appears to improve the outcome of patients with mucormycosis. Moreover culture results and/or histologic diagnosis are indispensable for programming secondary prophylaxis with amphotericin B or liposomal amphotericin, because Mucor is not responsive to azoles [14]. The outcome, however, remains critical: in hematologic neutropenic patients, mucormycosis is frequently characterized by disseminate disease and a rapidly fatal course.

This case shows the importance of an integrated and experienced team in the management of complex oral condition in medically complex patients. In addition to improving patient outcomes, early detection and successful management are critical in reducing the complications, which can drive the cost of care even higher. On the other hand, prevention and early stage diagnosis reduce dramatically the cost of care and improve patient's outcome. An integrated team facilitates detection, diagnosis and management of a potentially fatal fungal infection.

Conflict of interests: The authors declare no potential conflict of interests.

\section{References}

1. Lanternier F, Sun HY, Ribaud P, Singh N, Kontoyiannis DP, et al. (2012) Mucormycosis in Organ and Stem Cell Transplant Recipients. Clin Infect Dis 54: 1-8.

2. Spellberg B, Edwards J Jr, Ibrahim A (2005) Novel perspectives on mucormycosis: pathophysiology, presentation, and management. Clin Microbiol Rev 18: 556-569.

3. Sinko J, Csomor J, Nikolova R, Lueff S, Krivan G, et al. (2008) Invasive fungal disease in allogeneic hematopoietic stem cell transplant recipients: an autopsy driven survey. Transpl Infect Dis10: 106-109.
4. Petrikkos G, Skiada A, Lortholary O, Roilides E, Walsh TJ, et al. (2012) Epidemiology and clinical manifestations of mucormycosis. Clin Infect Dis 54: S23-S34.

5. McDermott NE, Barrett J, Hipp J, Merino MJ, Richard Lee CC, et al. (2010) Successful treatment of periodontal mucormycosis: report of a case and literature review. Oral Surg Oral Med Oral Pathol Oral Radiol Endodont. 109: e64-e69.

6. Morrison VA, McGlave PB. Mucormycosis in the BMT population. Bone Marrow Transplant.1993. 11:383-388.

7. Kontoyiannis DP, Marr KA, Park BJ, Alexander BD, Anaissie EJ, et al. (2010) Prospective surveillance for invasive fungal infections in hematopoietic stem cell transplant recipients, 2001-2006: overview of the Transplant-Associated Infection Surveillance Network (TRANSNET) database. Clin Infect Dis 50: 1091-1100.

8. Metzen D, Bohm H, Zimmermann M, Reuther T, Kübler AC, et al. (2012) Mucormycosis of the head and neck. J Craniomaxillofac Surg 40: e321e327.

9. Skiada A, Rigopoulos D, Larios G, Petrikkos G, Katsambas A (2012) Global epidemiology of cutaneous zygomycosis. Clin Dermatol 30: 628-632.

10. Al Akhrass F, Debiane L, Abdallah L, Best L, Mulanovich V, et al. (2011) Palatal mucormycosis in patients with hematologic malignancy and stem cell transplantation. Med Mycol 49: 400-405.

11. Skiada A, Lanternier F, Groll A, Pagano L, Zimmerli S, et al. (2013) Diagnosis and treatment of zygomycosis: guidelines from the 3rd European Conference on Infections in Leukemia (ECIL 3). Haematologica 98: 492-504.

12. Liat AH, Bilavsky E, Avitzur Y, Amir J (2010) Successful treatment of cutaneous zygomycosis with intravenous amphotericin B followed by oral posaconazole in a multivisceral transplant recipient. Transplantation 90:1133-1135.

13. Epstein JB, Kupferman SB, Zabner R, Rejali A, Hopp ML, et al. (2016) Early diagnosis and successful management of oral mucormycosis in a hematopoietic stem cell transplant recipient: case report and literature review. Support Care Cancer 24: 3343-3346.

14. Kontoyiannis DP, Lewis RE (2011) How I treat mucormycosis. Blood 118:1216-1224.

15. Nosari A, Oreste P, Montillo A, Carrafiello G, Draisci M, et al. (2000) Mucormycosis in the hematologic malignancies: an emerging fungal infection. Hematologica. 85: 1068-1071.

16. John BV, Chamilos G, Kontoyiannis DP (2005) Hyperbaric oxygen as an adjunctive treatment for zygomycosis. Clin Microbiol Infect 11: 515-517. 